\title{
Urgences
}

\section{Un " Monsieur " nommé " Désir "}

\section{Paul Chanel Malenfant}

Numéro 23, avril 1989

Lisière du livre

URI : https://id.erudit.org/iderudit/025513ar

DOI : https://doi.org/10.7202/025513ar

Aller au sommaire du numéro

Éditeur(s)

Urgences

ISSN

0226-9554 (imprimé)

1927-3924 (numérique)

Découvrir la revue

Citer cet article

Malenfant, P. C. (1989). Un " Monsieur " nommé " Désir ". Urgences, (23), 26-35.

https://doi.org/10.7202/025513ar

Ce document est protégé par la loi sur le droit d'auteur. L’utilisation des services d’Érudit (y compris la reproduction) est assujettie à sa politique d'utilisation que vous pouvez consulter en ligne.

https://apropos.erudit.org/fr/usagers/politique-dutilisation/
Cet article est diffusé et préservé par Érudit.

Érudit est un consortium interuniversitaire sans but lucratif composé de l’Université de Montréal, l’Université Laval et l’Université du Québec à Montréal. Il a pour mission la promotion et la valorisation de la recherche. https://www.erudit.org/fr/ 


\section{PAUL-CHANEL MALENFANT Un «Monsieur» nommé «Désir»}

\section{D'un livre blanc: (c)ouverture}

D'abord décrire le livre: un livre blanc. Ainsi se désigne d'emblée telle scénographie du scriptural puisque cette page blanche de couverture - apparaît bien, entre toutes les autres et au même titre qu'elles, comme un lieu d'inscription de la signifiance; en ce sens les signes (les seuils) graphiques et photographiques qui s'y donnent à lire sont d'entrée de jeu, littéralement comme scéniquement, constitués en texte. En outre, cette "couverture» blanche où s'expose une photographie - en noir et blanc - de l'auteur, portant de ce fait chemise blanche, pantalon et chaussures noirs, exhibe ainsi, en une sorte de stratégie déviée de strip-tease et par l'efficacité de l'ironie, sa discrète fonction d'«habillage» ou de livrée: particulièrement en un livre où se thématise, avec euphorie, les incontinences du déshabillage: "quand / je me déshabille je sais que je me / déshabille, c'est moderne (peut-être), im- / pétueux et impénitent, je n'ai pas vos petits / yeux pour vos masturbations coupables" (p. 31). Cette insistante mise à nu (histoire de célibataires, on verra...) énoncera d'ailleurs un peu plus loin son équivalence mystique et parodique: «quand je me / déshabille c'est une vraie religion" (p. 47). En l'espace de cette première page donc, via la photographie d'auteur et la superposition mimétique du noir sur blanc, s'inscrit la connivence du scripturaire et du vestimentaire. Dès lors tous les dispositifs concourront à multiplier les allusions spéculaires, par référence conjointe au visuel, au textuel et au sexuel comme à rappeler le travail simultané de productionexpression qui articule les «aventures" de l'écriture de ce livre: «de quel côté l'aventure nous déposera / entre autres? Ce ne sera pas une histoire d'a- / mour, ça bien été une histoire d'amour» (p. 16). On verra bien.

\section{Les herbes grises}

Ainsi sur cette couverture blanche (drap, lit ou livre, tous motifs inhérents à la poétique de l'auteur) sont disposés, dans l'ordre de la lecture linéaire, d'abord le nom de la maison d'édition inscrit en gris assez soutenu: "les herbes rouges". En même temps que cette couleur synthétise, comme par alliance d'éditeur à auteur, le camaïeu blanc-noir-gris de la photographie, le surplomb du titre éditorial, qui constitue le logo de la revue et, par consé- 
quent, son nécessaire emplacement, tendrait cependant à subordonner subrepticement l'instance auctoriale rendue pourtant très visible par l'effet de sa représentation photographique. S'instaurent donc ici, par préséance spatiale, à la fois une distanciation et une accointance: par apparentement et effet de solidarité, le nom métaphorique de l'éditeur relève du même registre coloré que la photographie de l'auteur; par priorité, la signification de l'onoma éditorial domine la désignation implicite dans le nom et la photographie. Se trouve ainsi posée, éditorialement, en cet apparent discord du générique paratextuel, la primauté de l'appareil producteur dont toute la fabrique de ce livre témoigne qu'elle pourrait relever du labeur conjoint de l'éditeur et de l'auteur (qui a d'ailleurs aussi réalisé la maquette de la couverture) au compte de qui pourrait être portée l'assertion: «il travaille en pleine obscurité, tout ce qui aura un nom, une surface, il est grand temps d'être impressionné» ( $p$. 4). D'où, redoublement encore, son «impressionnante» impression sur la pellicule, sur le livre, d'où, en tels lieux, l'inscription de sa productive présence visuelle.

\section{«rose cochon»}

Viennent ensuite, en contraste du gris au rose, le nom de l'auteur "André Roy» (sur une ligne) et le titre "Monsieur Désir» (sur deux lignes). Certes le choix de ce rose qui tient à la fois du póurpre ou du violet dilués (ou d'une sorte de rouge «passionnel»», puisqu'il s'agit ici, est-il dit sur la quatrième de couverture, du «Dernier cycle des «Passions"', dérivé de celui que dit, textuellement, l'oxymoron éditorial?) induit le procès même qui conduit de la coloration à la connotation. En effet, outre les multiples suggestions synesthésiques conventionnelles (rose bonbon, rose pour les ongles, rose pour les filles, roses de la passion, couleur chair, faire fleur de rose, etc.) qui s'associent assez spontanément à cette couleur, le texte en justifiera lui-même le choix paratextuel en accord avec les isotopies sexuelles qu'il met en scène: «mais que fais-tu derrière / mon dos? des sauts, des délices? alors que / je jubile, fabule pour des conduites / étonnantes, réussies, roses au goût” (p. 29). On reviendra sur la portée de cette teinte sexuelle à l'endos du livre et "derrière (le) dos" de l'auteur photographié, prolongé en quatrième de couverture, mais il importe encore de souligner le pertinence érotique de ce rose (éros, justement) sur la palette libidinale de Monsieur Désir de même que son impact sémantique sur la scène du déshabillage: «quand je me / déshabille c'est une vraie religion, et je fais / exprès pour être silencieux, consonnes à mes fesses, / rose cochon» (p. 47). Enfin, la réso- 
nance acoustique de ce rose (de «consonnes» à "cochon») se répercutera dans le motif, aussi sexuellement connoté, de la cloche: «nous / nous embrassions dans l'aube qui / clochait rose» (p. 46).

\section{Une “revue» sexy: le gai savoir}

Passage du gris au rose. Deux tracés roses figurant les lignes doubles d'une voie ferrée pourraient convoquer, traces transculturelles et via les échos titulaires de Monsieur Désir: «Un tramway (aussi) nommé désir». Cette allusion relèverait tant de la culture cinématographique de l'auteur que de telles autres incidences "spectaculaires» et spéculaires voire médiatiques, lisibles dans l'appareil paratextuel. En ces rails donc qui, contenant la photographie, le nom de l'auteur et le titre du livre, forment cadre sans rebords pour se poursuivre sur la quatrième de couverture, apparaît, en symétrie spatiale et colorée avec «les herbes rouges», le numéro de livraison de la revue: «88-89». II s'agit donc, d'une part, d'un numéro double de la revue publiée chez les frères Hébert, cet effet de redoublement confortant encore, même par coïncidence, le foisonnement des dispositifs de duplication et de réfraction qui caractérise simultanément, on le voit, la mise en texte et la mise en livre de Monsieur Désir. D'autre part, en ce contexte et élargissant le champ sémantique du "numéro de revue», aidé en cela par le sensationnalisme concerté du titre, «Monsieur Désir», le lecteur (spectateur et voyeur) pourrait se prendre à parcourir telle "revue» de gaies «folies bergères", mieux, à s'attarder devant une suggestive affiche de cinéma ou de spectacle de variétés sur laquelle clignoteraient en rose le nom de la star et le titre de sa performance. Ce travertissement discret qui conduirait de la «folie» à la «folle de nuit», de la posture «gay» à la «gaieté» - «mais qu'est-ce qu'être gai?» (p. 14) -, outre qu'il s'inscrit déjà dans le relai translinguistique qui va de Roy, nom propre et nom d'auteur (d'autorité) à "queen» (appellation de puissance monarchique), sera bien entendu, selon cette allusive cohérence mnémotechnique qui accorde les signes du texte et les seuils (même absents) du livre, littéralement textualisé et sexualisé: "les peaux dans la musique, les délicieuses, / les folles parce que regorge la nuit» (p. 33). Enfin, sur cette voie et en dépit des contingences éditoriales de la numérotation, ce numéro «88-89», dont la valeur symbolique habituelle donnerait à voir la spirale ou le tracé spéculaire (la boucle réverbérante) ne pourrait-il pas aussi s'entendre comme une manière d'analogon du sexuel ouroboros postural: «69»?. 
De ces dispositions graphiques et visuelles qui induisent en premier lieu une «lecture à vue» de Monsieur Désir, celle de la photographie de l'auteur est sans doute la plus significative. D'une part elle tient de la représentation figurative et elle accuse ainsi les tractations mimétiques qui ont cours, de l'objet-livre à l'effet de réel: "histoire d'être représentable, / je sais que je suis dans l'imitation générale» (p. 21). D'autre part, les neuf-dixièmes de la photographie occupant, côté gauche, la face recto du livre et l'autre dixième se trouvant replié en une étroite bande, côté droit, sur sa face verso, cette photographie attire ainsi l'attention par sa position de centre décentré et elle ajuste de façon assez efficacement clandestine, nous le verrons, le propos textuel (celui de la fiction) au sujet sexuel du livre: «disons pour faire fiction: du / poil, de belles fesses qui ont perdu / leur prosaïsme, que je me nommerai le chroniqueur / des impudeurs, des métaphores de la passion et / surtout le monsieur délicat aux éternelles / histoires de cul» (p. 40). Qu'en est-il donc de ce «ça bien été une histoire d'amour» (p. 16)?

Sur la section principale, en superficie, de la photographie qui apparaît côté recto du livre, l'auteur se trouve scindé, en la position de semi-accroupissement où il est, par une ligne, celle de l'épine (ou dos) du livre, tirée du bas de la colonne vertébrale (du dos) au galbe du postérieur. Cette ligne rencontre ainsi le corps d'A.R. (André Roy) à la jointure de la chemise et du pantalon (du blanc et du noir, donc) de la même manière que le titre, M.D. (Monsieur Désir), rencontre, linéairement, le corps d'A.R. (André Roy) au niveau (et en bas) de la ceinture. Pour assez sophistiquée qu'elle soit, en son ingénieuse concertation, cette occupation de l'espace scénique de la page de couverture engage de raffinés effets d'inversion (visuelle et sexuelle) et de suggestion libidinale. En effet, par un parcours vertical, le A de André s'inverse, figurément, en le M de Monsieur, tandis qu'un circuit horizontal induit l'inversion du D de Désir en cette forme de demi-disque noir (ce D), dont nous verrons qu'elle est, sur la quatrième de couverture, la coupe du postérieur de l'auteur photographié. Ainsi se donne la «tenue vestimentaire» d'un livre qui sollicite l'oeil du verso (vers et éros conjugués) à l'envers (au "recto" ou à son icône anatomique) et dont la lecture se prête sans cesse à la réversibilité (au sousentendu) des vers.

La pose détaillée du sujet photographique pourrait encore se définir ainsi: le bras droit, appuyé sur le genou, se prolonge d'une main fermée qui recouvre la bouche juste sous la moustache (du 
poil...), geste accordé à la posture conventionnelle d'un «penseur»; la coiffure est négligemment «clean»; le bras gauche porte aussi sur le senestre genou recourbé et le long index de la main pointe vers le bas de l'espace photographique, semblant à la fois désigner tel substitut phallique et orienter l'oeil du regardeur vers la partie arrière de la photographie; le regard de l'auteur paraît aigu sous des sourcils fournis: "dis-moi que sans mes verres fumés mes / yeux seraient une orgie» (p. 33); enfin, les pieds pointés par des talons relevés, l'apparent vernis des chaussures (pantoufle de verre / vair de Cendrillon, par référent intertextuel? ou illustration fétichiste de tels vers: pointés / pointus / luisants?) de même que toute la pose qui semble prise dans le suspens intermédiaire d'un élan, pourraient appartenir, compte tenu de l'écho porté par Les passions du samedi (1979) et le Petit supplément aux passions (1980), au «look» d'un John Travolta sage, réfléchissant momentanément à l'inextinguible «Fièvre du samedi soir".

\section{Photo - verso}

Tournons le livre. A la quatrième de couverture, la mince bande de photographie repliée par l'épine montre, comme en ombre portée sur le blanc, ce galbe noir d'un postérieur - ou d'un ovale: cul coupé! - qui constitue, on l'a vu, la pièce manquante du recto photographique et auquel l'isolement au verso confère une dimension non figurative de la même manière que la prise de vue partielle ou le gros plan tendent à abstraire l'objet représenté. "Désormais ce ne sera plus un cul, / on espère mais c'est ambigu» (p. 9). Ainsi se redit encore toute l'équivoque de la figuration et de l'imitation cachées.

En outre, ce signifiant visuel partiel (ou petit a... anus?!) est assorti, comme il a été dit, du sur-titre cumulatif («Dernier cycle des "Passions"») qui se trouve inscrit en rose sur blanc, à l'instar du nom de l'auteur et du titre sur la page de couverture, de ce même "Consonnes à mes fesses, / rose cochon" (p. 47), ici surdéterminé par la connivence avec l'image (il est bien «derrière(le) dos» de l'auteur) et par une présence accrue de cette couleur étalée au haut et au bas de la page sur les deux bandes qui délimitent maintenant l'espace blanc. De surcroît, allié à l'efficace du support visuel, le référent cyclique ultime du syntagme titulaire convoquera d'autres livres antérieurs d'André Roy, publiés chez le même éditeur. En ce sens, si le cul est bien, dans Monsieur Désir, le "Dernier cycle des "Passions" », lieu des tractations passionnelles certes, mais encore lieu des passages (des passades) sexuel(le)s, 
sa mise ${ }^{2}$ en scène visuelle et textuelle confédère tour à tour En image de ça (1974) (ça ${ }^{3}$ : de la chose et des mots pour la dire...), D'un corps à l'autre (1976), Corps qui suivent (1977), Le Sentiment du lieu (1978), et, bien sûr, l'ensemble du «Cycle des passions", là où passionné ça passe et fait des passes, bref tout ce circuit cyclothymique du corps à corps qu'explore l'oeuvre de Roy, toute cette drague intra et intertextuelle qu'elle exerce à plaisir, à désir. Et cette solidarité qui advient ici entre les textes comme seul lieu pour les passions, est dite d'un trait en l'incipit et l'explicit de Monsieur Désir: «Lieu très encourageant (...) j'suis seul» (p. 4 et 55).

\section{La lettre du désir: le désir de la lettre}

La contamination osmotique du sens entre les divers supports paratextuels de même que l'immédiate efficace de leur portée graphique et photographique à l'égard du propos proprement textuel engagent encore la filature de toute une spécularité du mot à mot et de la lettre, disposés aux abords du livre; s'y exaltent aussi, avec ce même cynisme thérapeutique, à la fois la narcissique phase du miroir ainsi que la jouissance étymologique et onomastique dont on sait qu'elle est une forme d'équivalence de la curiosité génétique: “Tous nés de la même langue"» (p. 27), tel est d'ailleurs, après “"Et vous m'appellerez Monsieur Désir"», le premier titre complémentaire de la deuxième partie du livre.

Ainsi dit encore le texte: «mon corps est là, mon nom, las, je le regarde, le / moment et la formule à fixer, à affiner, tout et / rien ne doivent m'échapper, c'est comment dire / et traduire "Je voudrais t'aimer" quand c'est un / cul, juste un" (p. 9). Et encore: "On est déjà peu sûr à moins de décliner son / nom» (p. 12). A la faveur de telles invites métatextuelles, observons comment les noms jouent sur la plage le jeu de l'essaimage kaléidoscopique et de la déclinaison permutative. On a déjà vu comment deux tracés roses encadraient (en miroir, en passe-partout) la photographie en noir et blanc de l'auteur, son nom et le titre du livre aussi inscrits en rose. A l'instar de tels autres effets issus de correspondances visuelles, l'emplacement des mots et des lettres frappe ici encore par insistante symétrie. D'abord le débordement, sur le gris photographique, du $\mathbf{A}$ rose de André et du $\mathbf{M}$ rose de Monsieur advient comme une sorte de signature de la photographie: ce Monsieur photographié c'est bien André Roy, c'est bien Monsieur Désir, titre en quelque sorte choisi comme surnom, comme pseudonyme assorti au nom, bref comme nom de plume; l'instance d'identification, de 
surcroît, se trouve renforcée par l'identité numérale des syntagmes onomastique et titulaire, par la symétrie des majuscules, enfin par l'identité numérique et l'inversion rimale des mots "Andrée" et "Désir».

Cette inclusion du littéral sur le photographique, qui relève simultanément d'une manière d'autographe et de dédicace, incline encore à «fixer» l'attention sur les interférences magnétisées par telles lettres et elle autorise maints ludiques (ou lubriques) déplacements. Voyons l'espace de la lettre:

les herbes rouges

André Roy

Monsieur

Désir

88-89 (en gris)

(en rose)

(en rose)

(en rose)

(en gris)

D'abord, la coïncidence spatiale du $\mathbf{A}$ et du $\mathbf{M}$ sur le rebord photographique induit toute une circulation verticale qui va de $« A$ Monsieur Désir», "A Mon Désir», «A Mon Dé» (celui du hasard, bien entendu...), «A Mon sieur», "A Mon sir» et, par remontée onomastique et généalogique (typographique aussi, puisque l'oeil remonte en "amont»), "A Mon Roy», tous énoncés qui pourraient relever de la plausible dédicace et prendre une valeur expressive accrue si tel lecteur voulait entendre en ce A dédicataire tout l'affect d'un Ah!

Une fois observée cette puissance de réfraction (et d'effraction) par l'intermédiaire de ce A -, première lettre du nom de l'auteur et de l'alphabet - toutes permutations sont permises, de l'endroit (l'And(ré) Roy) à l'envers (des vers à l'envers... comme le dit des pages, la chanson célèbre.... " «j'ai laissé tomber un vers" (p. 33)), jusqu'à l'envers du livre («André») rimant avec «Dernier» et «Dernier» rimant avec... «derrière») et ainsi du derrière au dessus de la couverture. Ces tractations paragrammatiques pourraient encore s'accroître du recoupement vertical des vocables «rouges» «[...]dré» - «Roy» - «[...]sieur» (celui-ci porteur acoustique de ce rieur qui partout s'esclaffe en ce livre...) - “Désir» (condensé sonore de «Monsieur" et "André») et des chiffres 88-89, liste où culminerait clandestinement l'ironie de la rougeur honteuse ou "coupable" (comme on dit conventionnellement de la verdeur...) du propos et de sa couleur atténuée, le rose. 
Ce qui se dit ici au niveau de la lettre et de l'irradiation du mot à mot, témoigne certes de la concertation paratextuelle comme instance de production du sens, mais encore ce dire s'active en divers lieux du livre. Ainsi, par exemple, les suggestions polysémiques déjà lisibles sur la page couverture se condensent en une autre formule sur la page de titre:

\section{André Roy}

\section{Monsieur}

\section{Désir}

Alors que le décalage des lettres par inclusion sur la photographie induisait tantôt la réversibilité d'une lecture pointilliste - «Justement je suis réversible, une pièce / instrumentale comme la fourrure / (ou la peinture)» (p. 50) -, ici, l'alignement triptyque en syllogisme inclinerait à détecter toutes les variantes de la permutation paradigmatique. André Roy c'est bien "Monsieur Désir» (comme on dirait «Monsieur Muscle»); mais encore, une fois le substantif transmué en verbe, la lecture s'active: André Roy désire Monsieur; André désire Monsieur Roy; Monsieur désire André Roy, etc. La polysémie tour à tour gaiement narcissique ou interpellative (incluant, bien entendu, l'obséquieuse formule domestique de la présentation distante et de l'offre de service: "Je suis André Roy. Monsieur désire quelque chose? quelqu'un?") qui ressort de ce vertige réverbérant trouvera son lieu de culmination synthétique dans le titre de la deuxième partie du livre: «Et / vous m'appellerez / Monsieur Désir», qu'il faudrait aussi entendre en cette traduction: Et vous m'appellerez (vous me nommerez, vous m'inviterez à venir...) André Roy. On aura observé, avec l'ironique voussoiement (ce vous de majesté) de l'interpellation plurielle, comment ce titre, autre effet de double, constitue encore une expansion éponyme de celui qui coiffe le livre. Enfin, parti pris de leitmotiv cette fois, il se répercute en l'explicit du texte: «vous m'appel- / lerez Monsieur Désir depuis / quelque temps, j'suis seul» (p. 55). Et cette solitude, en vertu de tout le système circulatoire de la signifiance qui active ce texte, engagera à son tour le motif du célibat qui semble aussi avoir présidé à la quête titulaire: «je lis le Grand Savoir du célibataire avec des / hoquets et des lapsus lascifs (je n'ai pas / encore trouvé de titre à ce recueil / que j'écris)" (p. 48). Et voilà que, réversibilité intertextuelle advenant (encore: «Justement je suis réversible, (...) comme la fourrure / (ou la peinture)» (p. 50) - le regardeur est désormais convoqué devant tel duchampien objet: «le marié (sic) mis à nu par ses célibataires, 
même", en vertu de la similitude des initiales qui fait ici se rencontrer Monsieur Désir et Marcel Duchamp.

\section{Le nom-lieu}

«Donc, à partir de ce moment d'autres dépla- / cements, royalement je m'approche, d'autres re- / gards, et nous en serions aux présentations, aux / ça-va?» (p. 11). A la jubilation onomastique manifeste en diverses instances paratextuelles s'adjoint donc, textualisée, la clandestinité toponymique; subrepticement le nom propre, "Roy», se transmue, "royalement", en une pose de dragueur - ( «dans la drague la main ouverte et le / regard précis; la réponse viendra comme un cou- / teau » (p. 6)) -, laquelle résorbe l'anonymat du lieu désigné par la première partie du texte: «Le parc / et la suite, / etc.» Ainsi ce «parc» où se trament, tout au cours du livre, les séductions et les guets apprêtés du désir - «'orientation utile pour la drague, ce parc» (p. 8); «le parc était venu et après» (p. 23); «Après: eh bien le parc» (p. 25); «l'émotion, la vue du parc» (p. 39) -, serait bien le parc du Mont-Royal: à lire enfin sans doute comme synecdoque du lieu conjoint des aventures et de l'écriture de ce texte: «Montréal, de nouveau à / Montréal il m'a confié ses images, ses / phrases» (p. 53).

\section{Le sens du détail, du décor, du désir}

Tout le système paratextuel de Monsieur Désir relève, on espère l'avoir montré, d'une rigoureuse volonté de mettre en scène le texte - «Tout est précis, tout est précis, ça fait im- / pression, voilà ce que je voulais dire: les désirs / ont encore une âme» (p. 10) -, de l'encadrer par un décor minutieux qui en soutienne l'écriture et lui serve en quelque sorte de contrepoint producteur: «nous sommes rigoureux même dans le désordre, cal- / culateurs comme techniciens; tu ne me liras pas à / ciel ouvert» (p. 23). Cet «apparat textuel», où tous les détails se concertent - «Le désir, le décor» ( $p$. 22) et «j'ai mon dictionnaire pour les / codes, et des désirs pour tous les jours» (p. 25) - de l'environnement au littéral, où la personne même qu'il explore, contribue de quelque manière à exciter le regard du lecteur et dès lors à engager le (dis)cours de la lecture. Car Monsieur Désir convie, à tout le moins dans les lieux du paratexte, par la présence même d'un corps dans le décor - "Je profite des détails de ton corps, / quelques idées (folles, perverses, elles deviennent / évidentes) » (p. 22) -, à L'espace de voir. Et de l'espace de voir au désir de lire un livre sur le désir, nulle distance. 
Car si, comme l'écrit Jean Starobinski, «Voir ouvre tout l'espace au désir, mais voir ne suffit pas au désir ${ }^{5}$, c'est bien une pulsion de désir (de plaisir) qui présidera à lecture du texte. Devant l'espace insuffisant (habillé) de ce qui est montré à l'oeil, de ce qui est donné à voir, le regard n'a pas d'autre issue que de pénétrer plus avant dans les pages, de lire ce qui du spectacle du texte le reconduit à son écriture, à sa mise à nu.

D'où l'esprit (et la lettre) de telle lecture: «certes / je deviendrai passionné.

/ Mais aussi nécessaire que /

la complicité, salut!» (p. 29)

1. «Le Cycle des passions» comprendra finalement quatre livres, le dernier étant Les lits de l'Amérique (1983). C'est en sous-titre à ce dernier livre qu'apparaît, dans sa graphie "définitive», le sur-titre général.

2. Cette mise au féminin renvoie, sur un autre plan, à la «mise» au masculin; en outre, elle prend tout son relief chez qui veut bien l'entendre à l'infinitif.

3. Ça: aussi rencontre des première et dernière lettres de «cinéma», lieu hautement évoqué et figuré en cette oeuvre.

4 Celle qui met en scène un autre roi: le roi Dagobert.

5. Jean Starobinski, L'oeil vivant, coll. «Le chemin", Paris, Gallimard, 1968, p. 13. 